\title{
Evacuation gastrique de l'orge et du blé chez le porc
}

\author{
J.P. LAPLACE, Odile PONS *, J.C. CUBER, Chantal KABORE \\ avec la collaboration technique de Claire CHABANET *, Brigitte MOUROT \\ et A. VAN HECKE \\ I.N.R.A., Laboratoire de Physiologie de la Nutrition \\ * Laboratoire de Biométrie \\ Centre de Recherches zootechniques, $F 78350$ Jouy-en-Josas
}

\begin{abstract}
Résumé
L'évacuation gastrique de l'orge et du blé broyés, simplement additionnés de minéraux et de vitamines ( 4 p. 100 de la ration), est étudiée chez 10 porcs de $49 \mathrm{~kg}$ équipés d'une canule gastrique permanente. L'étude cinétique est conduite par collecte des contenus gastriques résiduels après le repas à l'un des temps suivants : $30 \mathrm{mn}, 1,2,4$ ou $7 \mathrm{~h}$. Sur les digesta frais sont déterminés la matière sèche, l'amidon et l'azote. A raison d'une épreuve par jour, 3 cinétiques sont constituées pour chaque porc. La validité, dans le cas des céréales broyées, de l'usage de cette méthodologie, est établie par comparaison (fig. 2) avec les résultats obtenus après abattage (12 porcs supplémentaires) au temps $2 \mathrm{~h}$ après le repas.

Les quantités résiduelles dans l'estomac sont toujours plus importantes pour l'orge, quelle que soit la variable contrôlée (tabl. 3 et 4 ). L'analyse de variance multivariate confirme, en l'absence de tout autre effet significatif, le fait que l'évacuation gastrique du blé est au total plus importante que celle de l'orge (fig. 3). L'étude des courbes (ajustements polynomiaux) montre que cette différence est établie précocement, mais qu'ensuite le déroulement de l'évacuation entre $30 \mathrm{mn}$ et $7 \mathrm{~h}$ s'effectue selon la même cinétique pour les 2 céréales (parallélisme des courbes). Le facteur responsable de cet effet précoce ne peut être identifié a priori. Les résultats d'évacuation gastrique de l'amidon et de l'azote sont confrontés (fig. 4) aux données de la littérature concernant l'absorption des glucides et des acides aminés issus de la digestion de l'orge et du blé. Il apparaît que l'évacuation gastrique est largement déterminante de l'absorption des sucres réducteurs et des acides aminés; il n'est cependant pas possible de préciser si l'évacuation est ou non limitante de l'absorption.
\end{abstract}

Mots clés : Evacuation gastrique, céréales, porc, protéines, glucides.

\section{Introduction}

Les céréales telles que l'orge, le blé, ou le maïs constituent, à raison de 60 à 80 p. 100 , la base de l'alimentation du porc charcutier. L'importance quantitative de ces matières premières justifie l'étude comparative des conséquences de leur utilisa- 
tion à l'égard des bilans nutritionnels, des performances de croissance et de la composition corporelle des animaux (HENRY, 1968). Cependant, la digestion des céréales n'est connue que de façon très incomplète. Leur introduction dans le régime du porc en croissance nécessitant un apport complémentaire de matières azotées et d'acides aminés indispensables, l'attention a été principalement focalisée sur l'utilisation digestive de l'azote et de l'énergie et sur la disponibilité des acides aminés (BURACZEwSKA et al., 1979 ; Ivan \& Farrell, 1976 ; Low, 1979 a et b ; Sauer, Stothers \& PhilLIPS, 1977 ; SAUER et al., 1981). Encore faut-il noter que la plupart des informations disponibles concernent l'orge et le blé, céréales pour lesquelles a également été décrite (Rerat, Vaissade \& Vaugelade, 1979) l'absorption des sucres réducteurs et des acides aminés (apparition dans le sang de la veine porte).

Compte tenu du rôle non négligeable du rythme d'évacuation gastrique à l'égard de la cinétique de passage dans l'intestin et de la chronologie des processus de digestion qui s'y déroulent, il paraît intéressant de caractériser l'évacuation de ces céréales par l'estomac. On ne dispose en effet pour l'instant d'informations que pour des régimes à base d'amidons de maïs ou de blé purifiés, associés à diverses sources de protéines (LAPlace, Pons \& Simon, 1983 ; LAPlace et al., 1984). Mais aucune évaluation n'a été effectuée dans le cas des céréales entières présentées sous forme broyée.

L'objet du présent travail est donc l'étude comparative chez le porc de l'évacuation gastrique de l'orge et du blé broyés, céréales pour lesquelles on dispose par ailleurs du plus grand nombre d'informations.

\section{Matériel et méthodes}

Au total, 22 porcs mâles castrés de race Large White ont été utilisés. Dix d'entre eux, soumis à une fistulation de l'estomac, ont permis l'étude de l'évacuation gastrique de l'orge et du blé. Les douze autres porcs ont servi à l'évaluation, au cours d'un essai complémentaire recourant à la méthode des abattages, de la valeur absolue des mesures effectuées.

\section{A. Caractéristiques physiques et chimiques des aliments}

Les deux céréales ont été broyées au broyeur à marteaux (GoNDARD, TB 12) équipé d'une grille de $1 \mathrm{~mm}$. La granulométrie des produits ainsi obtenus a été déterminée à l'aide d'un appareil "Tamisor » équipé de six tamis à mailles carrées $(1,0$ $0,5-0,315-0,2-0,125$ et $0,08 \mathrm{~mm}$ ). Le temps de tamisage a été de $5 \mathrm{mn}$ pour chacun des trois échantillons de chacune des deux céréales. La distribution moyenne des tailles de particules est décrite par la figure 1.

Les aliments distribués aux porcs comportent 96 p. 100 d'orge ou de blé broyés et 4 p. 100 d'un complément minéral et vitaminique dont la composition a été décrite par HENRY \& RERAT (1966). Les principales caractéristiques chimiques des aliments ainsi préparés sont indiquées dans le tableau 1. 


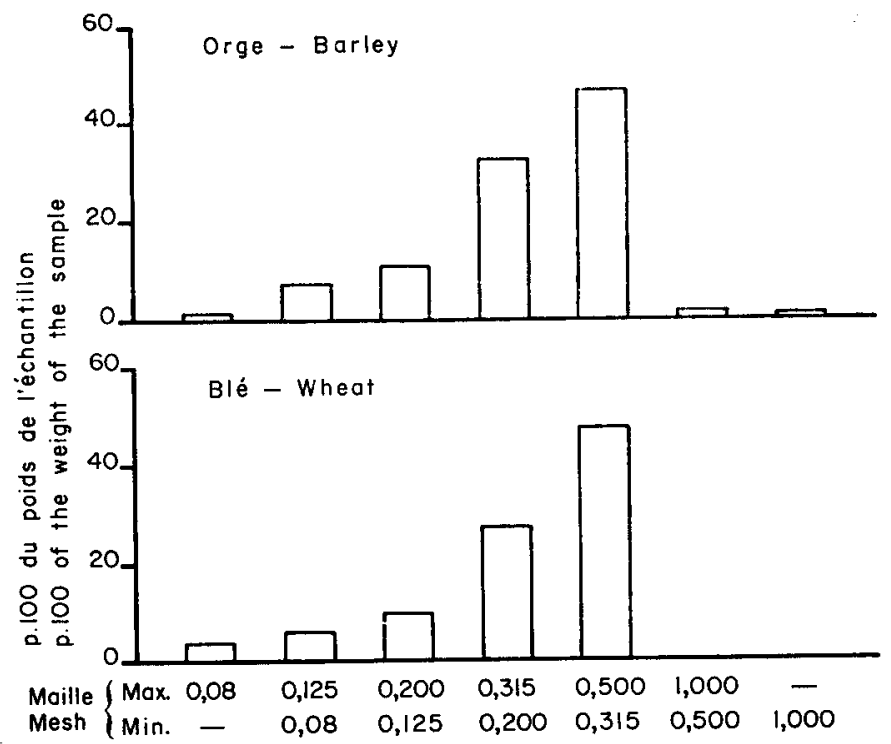

FIG. 1

Distribution moyenne des tailles des particules

résultant du broyage des deux céréales au broyeur à marteaux équipé d'une grille de $1 \mathrm{~mm}$. Mesures obtenues par séparation sur des tamis à maille carrée de $1,0-0,5-0,315-0,2-0,125$ et $0,08 \mathrm{~mm}$.

Mean distribution of particle size of the two cereals after hammer milling on $1 \mathrm{~mm}$ screen. Measurements on multiple screens with square mesh of $1.0-0.5-0.315-0.2-0.125$ and $0.08 \mathrm{~mm}$.

TABLEaU 1

Teneur en matière sèche (M.S.) des aliments à base d'orge ou de blé, et composition chimique (en p. 100 de la M.S.)

Dry matter (D.M.) content of the barley or wheat based diets, and chemical composition (per cent of D.M.)

\begin{tabular}{|c|c|c|}
\hline $\begin{array}{l}\text { Aliment } \\
\text { Diet }\end{array}$ & $\begin{array}{c}\text { Orge } \\
\text { Barley }\end{array}$ & $\begin{array}{c}\text { Blé } \\
\text { Wheat }\end{array}$ \\
\hline Matière sèche - Dry Matter ....... & 87,2 & 87,4 \\
\hline Amidon (1) - Starch $\ldots \ldots \ldots$ & 53,7 & 62,4 \\
\hline Azote (2) - Nitrogen $\ldots \ldots \ldots \ldots \ldots$ & 1,6 & 1,7 \\
\hline Cellulose brute (3) - Crude cellulose & 4,4 & 2,4 \\
\hline $\begin{array}{l}\text { Résidu neutre (4) } \ldots \ldots \ldots \ldots \ldots \ldots \\
\text { Neutral detergent fiber }\end{array}$ & 15,8 & 12,7 \\
\hline $\begin{array}{l}\text { Résidu acide }(4) \ldots \ldots \ldots \ldots \\
\text { Acid detergent fiber }\end{array}$ & 4,7 & 3,4 \\
\hline Lignine (4) - Lignin ..... & 0,7 & 0,8 \\
\hline Minéraux - Minerals ........... & 5,2 & 4,7 \\
\hline
\end{tabular}

(1) Cuber \& Laplace, 1979. (2) Kueldahl. (3) Weende. (4) Van Soest, 1963 a et b. 


\section{B. Alimentation et collecte des digesta chez les porcs fistulés}

Les dix porcs utilisés pour l'étude cinétique de l'évacuation gastrique de l'orge et du blé ont subi, à un poids vif de $48,6 \pm 0,8 \mathrm{~kg}$, une fistulation de l'estomac réalisée dans des conditions identiques à celles antérieurement décrites (CuBER, LAPlace \& Villiers, 1980). Ces 10 porcs, installés individuellement en cage à métabolisme, et affectés au hasard à l'une ou l'autre des 2 céréales à raison de 5 porcs pour chacune, ont été habitués aux aliments, orge ou blé selon le cas, durant 4 à 8 jours avant toute expérimentation. Ils reçoivent quotidiennement 2 repas à 9 et $16 \mathrm{~h}$, apportant chacun $1 \mathrm{~kg}$ de farine fraîche diluée par 1,51 d'eau (tabl. 2). La collecte des contenus gastriques est pratiquée dans tous les cas à la suite du repas du matin. Avant sa distribution, on procède au contrôle de la vacuité gastrique par abreuvement (500 $\mathrm{ml} \mathrm{d'eau),} \mathrm{la} \mathrm{canule} \mathrm{étant} \mathrm{ouverte.} \mathrm{Après} \mathrm{l'ingestion} \mathrm{du} \mathrm{repas} \mathrm{d'épreuve,} \mathrm{une}$ seule collecte des contenus gastriques résiduels est effectuée pour chaque jour d'expérience, à l'un des temps post-prandiaux ci-après : $30 \mathrm{mn}, 1 \mathrm{~h}, 2 \mathrm{~h}, 4 \mathrm{~h}, 7 \mathrm{~h}$. Les porcs reçoivent alors un repas de remplacement équivalant à la quantité collectée, sur la base d'une teneur moyenne en matière sèche des effluents gastriques de $30 \mathrm{p} .100$. Lorsque la collecte a lieu au temps $7 \mathrm{~h}$ après le repas d'épreuve, le repas de remplacement est fourni en une fois en complément du second repas quotidien (16 h). Dans tous les cas, une cinétique complète est établie en une semaine.

\section{TABleAu 2}

Quantités totales ingérées ( $g$ ) lors des repas d'épreuve de l'un ou l'autre des aliments à base (96 p.100) de céréales broyées.

Total quantities $(g)$ ingested as test meal according to the ground cereal based ( 96 per cent) diet fed to the pigs.

\begin{tabular}{|c|c|c|c|c|}
\hline $\begin{array}{l}\text { Céréale } \\
\text { Cereal }\end{array}$ & $\begin{array}{l}\text { Matière fraîche } \\
\text { Fresh mailer }\end{array}$ & $\begin{array}{l}\text { Matière sèche } \\
\text { Dry matter }\end{array}$ & $\begin{array}{c}\text { Amidon } \\
\text { Starch }\end{array}$ & $\begin{array}{c}\text { Azote total } \\
\text { Total nitrogen }\end{array}$ \\
\hline 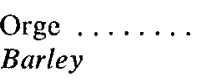 & 2500 & 860 & 462 & 13,46 \\
\hline $\begin{array}{l}\text { Blé } \ldots . . . \cdots \\
\text { Wheat }\end{array}$ & 2500 & 867 & 541 & 14,66 \\
\hline
\end{tabular}

11 a été prévu pour chaque céréale l'obtention de 75 mesures $(5$ porcs $\times 5$ temps $\times 3$ épreuves). Mais en pratique, selon la docilité des animaux, 13 à 17 mesures ont été réalisées pour chacun des 5 temps de la chronologie retenue et pour chacune des deux céréales. La durée moyenne de vie expérimentale des 10 porcs a été de 35,2 $\pm 2,3$ jours. Au cours de cette période leur gain de poids vif total a été en moyenne de $11,2 \pm 2,0 \mathrm{~kg}$.

\section{Essai complémentaire par la technique des abattages}

La méthodologie rappelée ci-dessus ne comporte pas de contrôle de la vacuité gastrique effective après la collecte des digesta par la fistule gastrique. Cette particu- 
larité évite toute manœuvre supplémentaire par rapport aux expériences antérieures (Cuber, laplace \& Villiers, 1980 ; Laplace, Pons \& Simon, 1983 ; Laplace et al., 1984). Pour ces dernières cependant, toutes relatives à l'évacuation de régimes semi-purifiés, il a été vérifié que la collecte par la fistule gastrique autorise effectivement une collecte totale (Cuber, Laplace \& Villiers, 1980). Etant donné la nature très différente (céréales broyées) des 2 régimes ici mis en ouvre, une série d'abattages a été réalisée pour évaluer une éventuelle sous-estimation des contenus gastriques résiduels dans l'hypothèse de leur écoulement incomplet par l'intermédiaire d'une fistule gastrique permanente.

Le poids vif moyen des 12 porcs utilisés pour ces abattages $(58,5$ - 1,3 kg) est analogue au poids moyen des porcs fistulés au terme de leur utilisation expérimentale $(59,8 \pm 1,2 \mathrm{~kg})$. Ces 12 porcs ont été hébergés en cage, dans des conditions identiques à celles offertes à leurs congénères. Ils ont été alimentés en deux repas quotidiens à $9 \mathrm{~h}$ et $16 \mathrm{~h}$ au cours des jours précédant l'abattage, soit avec le régime orge, soit avec le régime blé ( 6 porcs par céréale). Le repas du soir est supprimé la veille de l'épreuve. Pour celle-ci, le repas (orge ou blé selon le cas) est distribué à $9 \mathrm{~h}$ (tabl. 2) et l'abattage est effectué dans tous les cas au seul temps postprandial $2 \mathrm{~h}$.

Pour réaliser les mesures, l'animal est anesthésié à l'halothane dans sa cage, puis on pratique une laparotomie permettant de procéder au clampage du pylore et du cardia. L'estomac est prélevé rapidement dans sa totalité par section de l'œsophage abdominal, du duodénum proximal et de l'ensemble des mésos. L'animal est sacrifié cependant que l'estomac est essuyé avant ouverture. On procède alors à la récupération de la totalité du contenu gastrique résiduel à la faveur d'une incision de la grande courbure.

\section{Techniques analytiques}

Quel qu'ait été leur mode d'obtention (collecte ou abattage), les contenus gastriques sont pesés en l'état, homogénéisés vigoureusement et échantillonnés. Un premier échantillon d'environ $30 \mathrm{~g}$ est utilisé pour la détermination de la teneur en matière sèche (M.S.) par passage à l'étuve $\left(24 \mathrm{~h}\right.$ à $\left.104^{\circ} \mathrm{C}\right)$. Un second échantillon de même importance est prélevé puis lyophilisé en vue de la détermination de l'azote total (méthode Kjeldhal) et de l'amidon défini comme se référant au glucose et à tous les polymères $\alpha$ de ce dernier. L'amidon est dosé selon la méthode de HoLz (1977) modifiée par CuBER \& LAPLACE (1979). Ces diverses mesures permettent le calcul de la quantité totale de M.S. résiduelle dans l'estomac aux divers temps de collecte, ainsi que des quantités totales correspondantes d'azote et d'amidon.

\section{E. Analyse statistique des résultats}

Pour l'ensemble des essais (fistules et abattages), les quantités résiduelles moyennes de matière fraîche, matière sèche, amidon et azote enregistrées après ingestion de chacune des deux céréales, sont rapportées en pourcentage des quantités ingérées correspondantes. Pour une même variable et un même temps postprandial (porcs fistulés) ces valeurs moyennes font l'objet d'une comparaison par un test $t$ de Student. Enfin pour chaque variable, les valeurs moyennes enregistrées au temps $2 \mathrm{~h}$ chez les porcs fistulés et celles déterminées au même temps par abattage, font aussi l'objet d'une comparaison par un test $t$ de Student. 
Au-delà de cette première approche, une analyse des effets susceptibles de rendre compte des différences d'évacuation gastrique, observées chez les seuls porcs fistulés, est conduite sur les quantités évacuées ( $Q$ ingérée - $Q$ résiduelle) exprimées en grammes. Toutefois, l'analyse de variance ne porte, du fait des variations du nombre de mesures précédemment signalées, que sur 4 porcs (au lieu de 5 ) $\times 3$ cinétiques $\times 5$ temps, soit sur 60 mesures pour chacune des 2 céréales. Sur ce dispositif orthogonal l'analyse de variance est réalisée selon la méthodologie décrite antérieurement (LAPlace, Pons \& Simon, 1983). Le test des liens entre les mesures effectuées aux différents instants (test de la matrice de variance-covariance) fournit, pour les quatre variables étudiées, des valeurs de la statistique du rapport de vraisemblance conduisant (selon une loi de $\chi^{2}$ ) à rejeter l'hypothèse d'indépendance des données. Aussi est-il nécessaire d'utiliser un modèle multivariate constitué par 24 vecteurs données $(2$ régimes $\times 4$ porcs $\times 3$ cinétiques $)$ de taille 5 , c'est-à-dire comportant chacun les 5 mesures effectuées sur un porc pour constituer une cinétique en une semaine. La durée constitue donc une dimension du modèle et ne peut être prise en compte comme un facteur.

Le modèle retenu s'écrit :

$$
\overrightarrow{Y_{r p s}}=\left(a+b_{r}+I_{r p}+c_{s}+d_{r s}+J_{r p s}\right) 1, \ldots, 5
$$

Par rapport à une valeur moyenne (terme a), l'addition du terme $b_{r}$ introduit l'effet propre du régime. Le terme $\mathrm{I}_{\mathrm{rp}}$ rend compte de l'effet hiérarchique régime/ porc dans la mesure où, chaque animal étant assigné à un seul régime, l'effet aléatoire du porc est lié à l'effet du régime. Le terme $\mathrm{c}_{\mathrm{s}}$ exprime l'effet de la semaine de constitution d'une cinétique; en effet, si l'indépendance des mesures d'une semaine à l'autre est admise a priori, les cinétiques consécutives ne sont pas nécessairement d'authentiques répétitions. Le terme $\mathrm{d}_{\mathrm{rs}}$ correspond à l'interaction régime-semaine. La résiduelle $J_{r p s}$ introduit toute autre source de variation non contrôlée.

Pour chacune des variables étudiées et chacun des termes du modèle, l'hypothèse de nullité du facteur considéré est testée par une statistique qui suit une loi de Wilks. Le calcul permet d'obtenir une estimation des divers termes à partir de laquelle peuvent êtré construites des courbes moyennes d'évacuation exprimant tel ou tel des effets fixes du modèle. Eventuellement, l'influence du temps peut être approchée en procédant à l'étude de ces courbes par l'application du modèle de PotThof \& Rox (1964) pour choisir le degré du polynôme le mieux adapté pour décrire l'évacuation gastrique en fonction du temps.

\section{Résultats}

\section{A. Quantités résiduelles dans l'estomac}

\section{Porcs fistulés}

Les quantités résiduelles de matière fraîche collectées en moyenne aux 5 temps après le repas (tabl. 3) sont dans l'ensemble assez voisines. On note seulement dans le cas de l'orge une quantité résiduelle significativement supérieure à celle collectée 
pour le blé à $30 \mathrm{mn}(\mathrm{p}<0,001)$ et $2 \mathrm{~h}(\mathrm{p}<0,05)$. La variabilité des quantités recueillies, exprimée par le coefficient de variation, est analogue pour les 2 céréales au cours des quatre premières heures postprandiales. Elle est par contre très supérieure dans le cas du blé au temps $7 \mathrm{~h}$ (tabl. 3).

\section{Tableau 3}

Quantités résiduelles moyennes (en p. 100 des quantités ingérées) de matière fraîche et de matière sèche dans l'estomac aux 5 instants de mesure, selon la nature de la céréale.

Mean residual quantities (as percent of the corresponding ingested quantities) of wet and dry matter in the stomach at the five collection times, according to cereal.

\begin{tabular}{|c|c|c|c|c|c|c|c|}
\hline \multicolumn{3}{|c|}{$\begin{array}{l}\text { Temps après le repas d'épreuve } \\
\text { Time after the test meal }\end{array}$} & $30 \mathrm{mn}$ & $1 \mathrm{~h}$ & $2 \mathrm{~h}$ & $4 \mathrm{~h}$ & $7 \mathrm{~h}$ \\
\hline \multirow{6}{*}{$\begin{array}{l}\text { Matière fraiche } \\
\text { Wet contents }\end{array}$} & \multirow{3}{*}{$\begin{array}{c}\text { Orge } \\
\text { Barley }\end{array}$} & n. & 16 & 14 & 13 & 16 & 16 \\
\hline & & Q. & $\begin{array}{r}94,8 \\
\pm 1,6\end{array}$ & $\begin{array}{r}89,8 \\
\pm 1,8\end{array}$ & $\begin{array}{r}83,8 \\
\pm 2,6\end{array}$ & $\begin{array}{r}66,4 \\
\pm 4,1\end{array}$ & $\begin{array}{r}40,9 \\
\pm 2,4\end{array}$ \\
\hline & & C.V. & 6,9 & 7,5 & 11,1 & 24,7 & 23,5 \\
\hline & \multirow{3}{*}{$\begin{array}{c}\text { Blé } \\
\text { Wheat }\end{array}$} & $\mathrm{n}$. & 14 & 14 & 15 & 15 & 14 \\
\hline & & Q. & $\begin{array}{r}86,1 \\
\pm 2,6\end{array}$ & $\begin{array}{r}85,8 \\
\pm 1,3\end{array}$ & $\begin{array}{r}76,6 \\
\pm 2,4\end{array}$ & $\begin{array}{r}64,8 \\
\pm 4,1\end{array}$ & $\begin{array}{r}37,5 \\
\pm 3,5\end{array}$ \\
\hline & & C.V. & 5,7 & 11,1 & 12,0 & 24,6 & 34,6 \\
\hline \multirow{6}{*}{$\begin{array}{l}\text { Matière sèche } \\
\text { Dry matter }\end{array}$} & \multirow{3}{*}{$\begin{array}{c}\text { Orge } \\
\text { Barley }\end{array}$} & n. & 16 & 14 & 13 & 16 & 16 \\
\hline & & Q. & $\begin{array}{r}76,7 \\
\pm 1,8\end{array}$ & $\begin{array}{r}71,8 \\
\pm 1,1\end{array}$ & $\begin{array}{r}58,8 \\
\pm 1,2\end{array}$ & $\begin{array}{r}37,0 \\
\pm 1,6\end{array}$ & $\begin{array}{r}19,4 \\
\pm 1,7\end{array}$ \\
\hline & & C.V. & 9,3 & 5,9 & 7,4 & 17,6 & 34,2 \\
\hline & \multirow{3}{*}{$\begin{array}{c}\text { Blé } \\
\text { Wheat }\end{array}$} & n. & 14 & 14 & 15 & 15 & 14 \\
\hline & & Q. & $\begin{array}{r}75,3 \\
\pm 1,6\end{array}$ & $\begin{array}{r}64,6 \\
\pm 1,5\end{array}$ & $\begin{array}{r}52,7 \\
\pm 1,3\end{array}$ & $\begin{array}{r}36,0 \\
\pm 2,3\end{array}$ & $\begin{array}{r}14,8 \\
\pm 1,6\end{array}$ \\
\hline & & C.V. & 7,8 & 8,9 & 9,7 & 24,3 & 39,7 \\
\hline
\end{tabular}

$\mathrm{n}=$ Nombre de mesures - Number of data.

$\mathrm{Q}=$ Quantité moyenne \pm écart type de la moyenne - Mean quantity \pm s.e.m.

C.V. = Coefficient de variation (p. 100) - Coefficient of variation ( $p .100)$.

Dans le cas de la matière sèche, la décroissance des quantités moyennes résiduelles enregistrées aux 5 temps postprandiaux (tabl. 3) diffère sensiblement selon la céréale : les quantités résiduelles sont en effet significativement plus importantes dans le cas de l'orge aux temps $1 \mathrm{~h}(\mathrm{p}<0,001), 2 \mathrm{~h}(\mathrm{p}<0,005)$ et $7 \mathrm{~h}(\mathrm{p}<0,10)$. Le coefficient de variation des mesures est du même ordre de grandeur jusqu'au terme de la deuxième heure postprandiale. Il est plus élevé dans le cas du blé aux temps 4 et $7 \mathrm{~h}$ (tabl. 3). Enfin, la teneur en matière sèche des contenus gastriques recueillis, analogue pour les deux céréales, décroît régulièrement, de 28 à 30 p. 100 au temps $30 \mathrm{mn}$, à 13 à $16 \mathrm{p}$. 100 au temps $7 \mathrm{~h}$.

Les quantités résiduelles d'amidon ne sont dans l'ensemble que faiblement supérieures dans le cas de l'orge (tabl. 4). La signification des écarts les plus importants 
par rapport au blé (à 1,2 et 7 h) reste limitée $(p<0,10)$. Les coefficients de variation des mesures sont encore analogues pour les 2 céréales à $30 \mathrm{~mm}, 1 \mathrm{~h}$ et $2 \mathrm{~h}$, et un peu plus importants pour le blé que pour l'orge à 4 et $7 \mathrm{~h}$ (tabl. 4).

\section{Tableau 4}

Quantités résiduelles moyennes (en p. 100 des quantités ingérées) d'amidon et d'azote dans l'estomac aux 5 instants de mesure, selon la nature de la céréale.

Mean residual quantities (as per cent of the ingested quantities) of starch and nitrogen in the stomach at the five collection times, according to the cereal.

\begin{tabular}{|c|c|c|c|c|c|c|c|}
\hline \multicolumn{3}{|c|}{$\begin{array}{l}\text { Temps après le repas d'épreuve } \\
\text { Time after the test meal }\end{array}$} & $30 \mathrm{mn}$ & $1 \mathrm{~h}$ & $2 \mathrm{~h}$ & $4 \mathrm{~h}$ & $7 \mathrm{~h}$ \\
\hline \multirow{6}{*}{$\begin{array}{l}\text { Amidon } \\
\text { Starch }\end{array}$} & \multirow{3}{*}{$\begin{array}{c}\text { Orge } \\
\text { Barley }\end{array}$} & $\mathrm{n}$. & 16 & 14 & 13 & 16 & 16 \\
\hline & & Q. & $\begin{array}{r}71,4 \\
\pm 1,7\end{array}$ & $\begin{array}{r}68,1 \\
\pm 1,6\end{array}$ & $\begin{array}{r}55,0 \\
\pm 1,8\end{array}$ & $\begin{array}{r}34,3 \\
\pm 1,8\end{array}$ & $\begin{array}{r}18,2 \\
\pm 1,9\end{array}$ \\
\hline & & C.V. & 9,7 & 8,7 & 11,8 & 21,4 & 40,9 \\
\hline & \multirow{3}{*}{$\begin{array}{c}\text { Blé } \\
\text { Wheat }\end{array}$} & $\mathrm{n}$. & 14 & 14 & 15 & 15 & 14 \\
\hline & & Q. & $\begin{array}{r}72,8 \\
\pm 1,6\end{array}$ & $\begin{array}{r}63,8 \\
\pm 1,6\end{array}$ & $\begin{array}{r}50,6 \\
\pm 1,5\end{array}$ & $\begin{array}{r}35,7 \\
\pm 2,5\end{array}$ & $\begin{array}{r}13,2 \\
\pm 1,6\end{array}$ \\
\hline & & C.V. & 8,1 & 9,3 & 11,8 & 27,6 & 46,2 \\
\hline \multirow{6}{*}{$\begin{array}{l}\text { Azote } \\
\text { Nitrogen }\end{array}$} & \multirow{3}{*}{$\begin{array}{c}\text { Orge } \\
\text { Barley }\end{array}$} & $\mathrm{n}$. & 16 & 14 & 13 & 16 & 16 \\
\hline & & Q. & $\begin{array}{r}74,1 \\
\pm 2,9\end{array}$ & $\begin{array}{r}69,1 \\
\pm 2,1\end{array}$ & $\begin{array}{r}53,5 \\
\pm 1,9\end{array}$ & $\begin{array}{r}31,8 \\
\pm 2,5\end{array}$ & $\begin{array}{r}13,5 \\
\pm 1,3\end{array}$ \\
\hline & & C.V. & 15,5 & 11,3 & 12,7 & 32,0 & 38,8 \\
\hline & \multirow{3}{*}{$\begin{array}{c}\text { Blé } \\
\text { Wheat }\end{array}$} & $\mathrm{n}$. & 14 & 14 & 15 & 15 & 14 \\
\hline & & Q. & $\begin{array}{r}65,1 \\
\pm 2,8\end{array}$ & $\begin{array}{r}55,0 \\
\pm 1,5\end{array}$ & $\begin{array}{r}44,9 \\
\pm 2,1\end{array}$ & $\begin{array}{r}26,2 \\
\pm 2,1\end{array}$ & $\begin{array}{r}10,7 \\
\pm 1,5\end{array}$ \\
\hline & & C.V. & 16,1 & 10,4 & 17,7 & 30,6 & 51,7 \\
\hline
\end{tabular}

Abréviations : voir tableau 3 - Abbreviations : see table 3.

Pour ce qui concerne l'azote, les quantités résiduelles recueillies dans le cas de l'orge sont significativement plus importantes que pour le blé jusqu'au temps $4 \mathrm{~h}$ (tabl. 4) : p $<0,05$ à $30 \mathrm{mn} ; \mathrm{p}<0,001$ à 1 et $2 \mathrm{~h} ; \mathrm{p}<0,10$ à $4 \mathrm{~h}$. Le coefficient de variation des mesures reste du même ordre de grandeur pour les 2 céréales jusqu'à $4 \mathrm{~h}$; il est beaucoup plus élevé dans le cas du blé au temps 7 h (tabl. 4).

\section{Porcs sacrifiés $2 h$ après le repas}

Les quantités de matière fraîche, matière sèche, amidon et azote, résiduelles dans l'estomac $2 \mathrm{~h}$ après le repas, et déterminées par la méthode des abattages, sont analogues à celles mesurées au même temps chez les porcs fistulés, quelle que soit la céréale ingérée (fig. 2). En effet, les quantités résiduelles de matière fraîche et d'azote déterminées par abattage ne diffèrent de celles recueillies chez les porcs fistulés que d'une quantité équivalant en moyenne à 3,3 à 4,1 p. 100 de l'ingéré correspondant. 


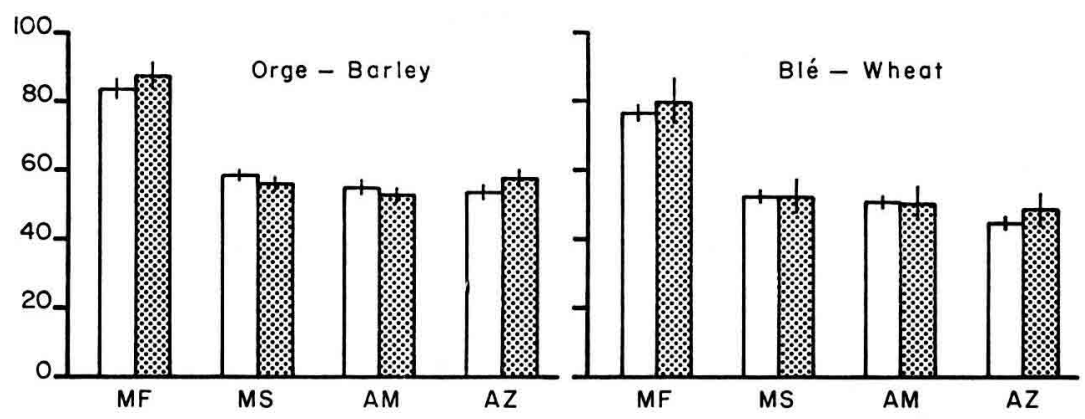

FIG. 2

Quantités résiduelles moyennes (en p. 100 des quantités ingérées) de matière fraîche (MF), matière sèche (MS), amidon (AM) et azote (AZ) dans l'estomac,

2 heures après ingestion d'un aliment à base $(96 \mathrm{p} .100)$ d'orge ou de blé broyés.

Mean residual quantities (as per cent of ingested quantities) of wet digesta (MF). dry matter (MS), starch $(A M)$ and nitrogen $(A Z)$ in the stomach, 2 hours after feeding a barley or wheat based $(96 \mathrm{p} .100)$ diet.

Colonnes blanches : collectes chez les porcs à fistule gastrique permanente (orge : $\mathrm{n}=13$; blé : $\mathrm{n}=15)$. Colonnes grisées : collectes par la méthode des abattages $(\mathrm{n}=6$ dans tous les cas). L'écart type de la moyenne est indiqué sur chaque colonne.

White columns : collection in pigs with a permanent gastric fistula (barley : $n=13$; wheat : $n=15$ ). Dark columns : collection by the slaughter method in intact pigs ( $n=6$ in all cases). The standard error of the mean is indicated for each mean value.

Les quantités de matière sèche et d'amidon ne s'écartent de celles déterminées chez les porcs fistulés que d'une quantité au plus équivalente à 2,5 p. 100 (M.S.) ou 0.7 p. 100 (amidon) de l'ingéré correspondant. Enfin le coefficient de variation des quantités recueillies lors d'abattage $2 \mathrm{~h}$ après le repas est, pour les 4 variables contrôlées, compris entre 8,5 et 10,6 p. 100 dans le cas de l'orge, et entre 23,7 et 25,7 p. 100 dans le cas du blé.

\section{B. Analyse de variance multivariate (porcs fistulés)}

L'analyse multivariate des effets susceptibles de rendre compte des différences d'évacuation gastrique au cours de la période postprandiale de 7 heures montre que, pour chacune des 4 variables considérées, l'hypothèse de nullité de l'effet du terme hiérarchique régime/porc, de l'effet de la semaine, et de l'interaction régime-semaine est acceptée sans ambiguïté (valeurs de la statistique très inférieures au seuil de rejet de l'hypothèse de nullité). En somme, il n'y a pas d'interférence aléatoire de l'animal et les 3 cinétiques constituées en 3 semaines consécutives sont d'authentiques répétitions, en l'absence d'effet propre ou d'interaction du facteur semaine.

Par contre, l'effet propre du régime - c'est-à-dire de la céréale broyée qui en constitue l'essentiel - est significatif pour les 4 variables contrôlées : l'hypothèse de nullité est rejetée au seuil 5 p. 100 dans le cas de la matière fraîche et à un seuil très inférieur à 1 p. 1000 pour la matière sèche, l'amidon et l'azote. Ceci revient à affirmer qu'au total, pour les 4 variables contrôlées, l'évacuation gastrique est plus 


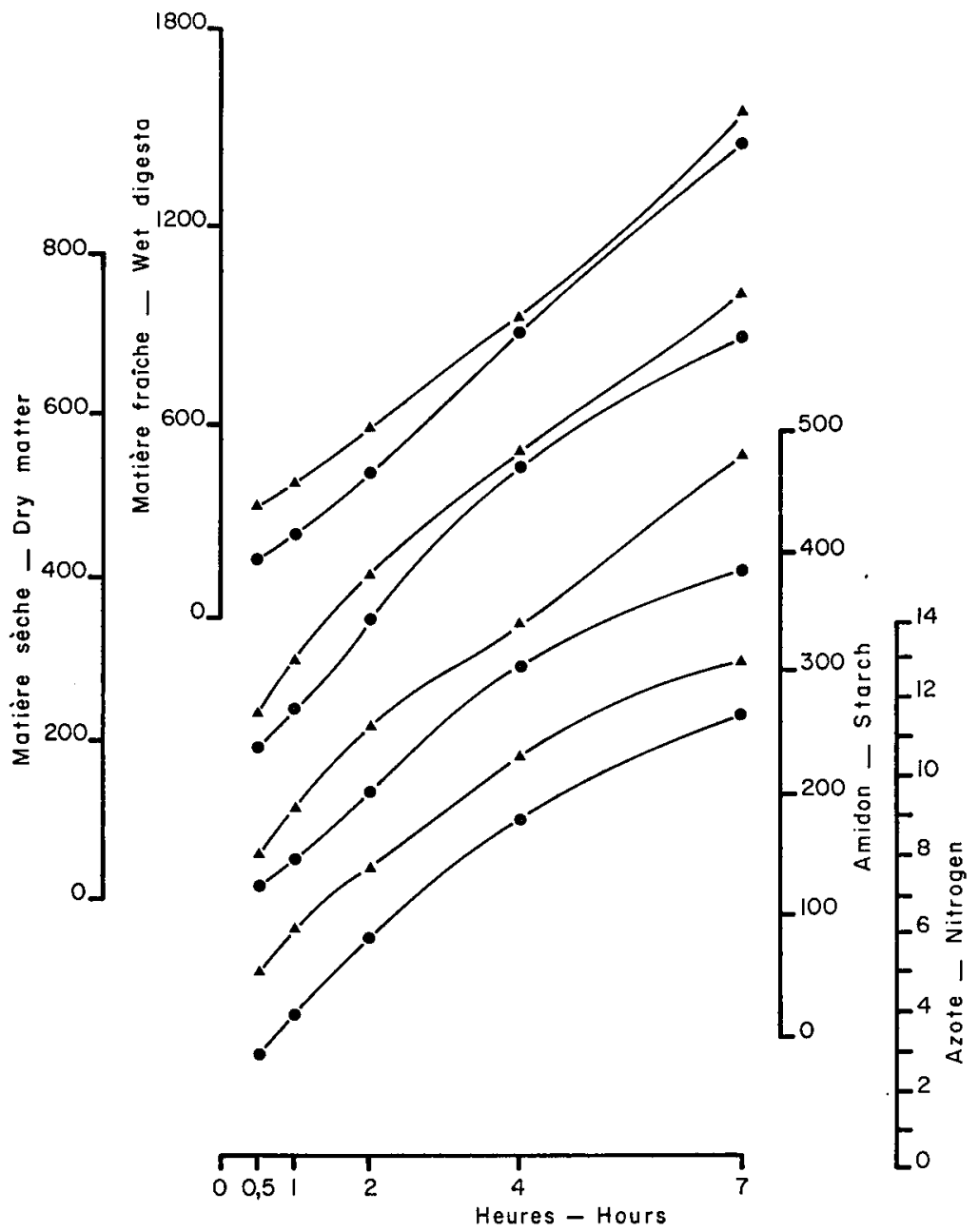

FIG. 3

Courbes construites par l'ordinateur (régressions polynomiales), qui expriment la quantité évacuée par l'estomac Y (grammes, en ordonnée) de matière fraîche, de matière sèche, d'amidon et d'azote, en fonction du temps $x$ (heures, en abscisse)

après ingestion d'un aliment à base d'orge (†) ou de blé (\) broyés.

Computer drawn curves (based on polynomial regressions)

representative of the quantity emptied from the stomach $Y$ (grams, ordinate) of either wet digesta, dry matter, starch or nitrogen,

as a function of the time $x$ (hours, abscissa) after intake of a diet based on ground cereals, either barley $(\bullet)$ or wheat $(\mathbf{\Delta})$.

rapide pour le blé que pour l'orge, comme en témoignent les courbes moyennes d'évacuation (fig. 3) obtenues par ajustement polynomial (degré maximum 4 compte tenu des 5 instants d'observation) ou reconstruites à partir du calcul des estimateurs pour l'effet fixe significatif du régime. Au-delà de cette différence globale, l'étude des courbes permet d'approcher l'influence du temps, c'est-à-dire le décours de l'éva- 
cuation, et d'apprécier d'éventuelles différences de la cinétique du phénomène au travers de tests de parallélisme. Il s'avère que pour chacune des variables contrôlées, le parallélisme est accepté entre les profils polynomiaux exprimant l'évacuation des deux céréales par un polynôme de degré 1 (matière fraîche, azote), 2 (amidon) ou 3 (matière sèche).

\section{Discussion}

\section{A. Evacuation comparée de l'orge et du blé}

L'analogie des bilans d'évacuation gastrique réalisés $2 \mathrm{~h}$ après le repas, par la technique des abattages et par la technique de fistulation gastrique chronique, constitue un élément important. Ce résultat témoigne du fait que la collecte des contenus gastriques résiduels par la fistule, telle qu'elle a été développée (CUBER, LAPLACE \& Villiers, 1980) et appliquée jusqu'ici à des régimes semi-purifiés (LAPlace, Pons \& Simon, 1983 ; LAPlace et al., 1984), est également utilisable pour des aliments de texture très différente tels que l'orge ou le blé broyés. La validité de l'étude effectuée chez les porcs fistulés se trouve donc établie dans la mesure où la collecte par la fistule est bien une collecte complète. Cette affirmation, basée sur les résultats de mesure au temps $2 \mathrm{~h}$, paraît pouvoir être étendue valablement à l'ensemble de la période postprandiale considérée. Par contre la validité des collectes par une fistule gastrique ne saurait être généralisée a priori à divers régimes complexes. La vérification apportée dans ce travail reste limitée aux cas considérés, orge et blé, sous les conditions de broyage précisées.

La variabilité de l'évacuation gastrique (exprimée par le coefficient de variation des mesures) est appréciée de façon différente selon la méthodologie mise en œuvre. Chez les porcs fistulés, le coefficient de variation des mesures pour une céréale à un instant donné prend en compte les résultats obtenus sur 5 porcs à raison de 3 essais par animal ; mais les termes $c_{s}$ et $I_{p p}$ sont considérés comme nuls par l'analyse multivariate. Lors d'abattage, le coefficient de variation concerne, pour une céréale, 6 porcs n'ayant fourni chacun qu'un seul essai ; or l'effet de l'animal est généralement très important (LAPlace, Pons \& Simon, 1983). On attend donc a priori une plus grande variabilité pour les mesures obtenues par la technique des abattages que lors d'usage de la fistulation. Ceci est vérifié dans le cas du blé pour lequel les coefficients de variation sont à peu près doubles dans le cas des abattages (23,7 à 25,7 p. 100) par rapport à la fistulation $(9,7$ à 17,7 p. 100 selon la variable contrôlée au temps $2 \mathrm{~h})$. Par contre, dans le cas de l'orge, l'ordre de grandeur des coefficients de variation reste le même pour toutes les variables quelle que soit la méthodologie mise en œuvre. L'origine de cette différence de répétabilité des mesures selon la méthodologie et la céréale considérées reste obscure. Par contre, la plus grande rapidité d'évacuation gastrique pour le blé que pour l'orge (porcs fistulés) répond très certainement des coefficients de variation sur les quantités résiduelles, qui sont à $7 \mathrm{~h}$ toujours les plus élevés dans le cas du blé. En effet la variabilité croît toujours au fil du déroulement de l'évacuation; elle est donc la plus forte pour l'évacuation qui est la plus avancée (cas du blé), particularité vérifiée pour chacune des quatre variables contrôlées.

Hors ces divers aspects méthodologiques, les résultats obtenus montrent sans ambiguïté que l'évacuation gastrique est quantitativement plus importante pour le 
blé que pour l'orge. Cependant, le parallélisme de leurs profils d'évacuation, quelle que soit la variable étudiée, implique que la distance entre ces profils tient à une ordonnée à l'origine différente, en l'absence de différence de cinétique dans le décours de l'évacuation. En somme, pour les 2 céréales, la quantité évacuée à $30 \mathrm{mn}$ s'incrémenterait d'une même quantité supplémentaire au cours du temps, l'évacuation se déroulant de la même façon au-delà de $30 \mathrm{mn}$. La question de savoir pourquoi le niveau moyen d'évacuation du blé est supérieur à celui de l'orge, ou encore pourquoi - en termes de bilan - la quantité évacuée à tel ou tel temps postprandial est plus grande pour le blé que pour l'orge, se résumerait alors à celle de connaitre les facteurs affectant l'évacuation précoce (avant $30 \mathrm{mn}$ ) de ces céréales. Dès lors l'analyse des profils d'évacuation au cours du temps postprandial n'a plus guère d'intérêt. De plus, il est bien difficile de comparer ces ajustements, obtenus pour des céréales entières broyées, apportant leurs propres constituants amylacés, azotés et membranaires, à ceux obtenus pour des aliments comportant un amidon purifié, une source de protéines d'origine végétale ou animale, et une cellulose de bois purifiée.

Il a été précédemment admis (LAPlace, Pons \& Simon, 1983) que les protéines joueraient un rôle décisif à l'égard de la chronologie de l'évacuation de la matière fraîche et de la matière sèche. Or si le degré de l'ajustement diffère entre céréales (degré 1) et régimes purifiés (degré 2) pour la matière fraîche et l'azote, ce n'est pas le cas pour la matière sèche (degré 3 dans tous les cas), malgré la différence qualitative et quantitative des matières azotées ingérées. Par ailleurs, le type d'association amidon-protéines s'est avéré (LAPlace, Pons \& Simon, 1983) influencer le niveau moyen d'évacuation de l'amidon et par voie de conséquence celui de la matière sèche, dans le cas de régimes purifiés. Mais rien ne permet de savoir si la différence d'évacuation moyenne de l'orge par rapport au blé tient plutôt à un facteur qualitatif spécifique tel que caractéristiques physico-chimiques de leurs amidons, composition en acides aminés de leurs protéines, ou nature de leurs constituants pariétaux, ou si elle relève de facteurs quantitatifs (orge plus pauvre en amidon et plus riche en constituants pariétaux que le blé), ou encore s'il s'agit d'une influence composite faisant intervenir conjointement ces divers facteurs potentiels qualitatifs et quantitatifs. Au total, il serait bien aléatoire de vouloir attribuer la différence enregistrée dans ce travail à un facteur particulier, a fortiori si l'on admet que celui-ci intervient dès les 30 premières minutes postprandiales. Il s'avère également difficile de transposer directement au cas d'un aliment complexe comme une céréale (qui n'est encore qu'une matière première pour des régimes usuels), les principes explicatifs définis sur une base analytique de régimes purifiés.

\section{B. Evacuation gastrique de l'amidon et digestion des glucides de l'orge et du blé}

Quels que soient en définitive le ou les facteurs explicatifs du fait que l'évacuation du blé est, à tout instant de la cinétique, plus importante que celle de l'orge, on peut s'interroger sur la signification de cette différence en termes de digestion et d'absorption. Dans le cas des glucides le rapprochement de nos observations avec les données relatives à l'absorption des sucres réducteurs après ingestion d'orge ou de blé (Rerat, Vaissade \& Vaugelade, 1979) peut être tenté, même s'il est nécessairement approximatif : l'évacuation de l'amidon est seule prise en compte alors que les céréales comportent d'autres glucides; l'absorption des sucres réducteurs n'est par ailleurs qu'une expression imparfaite de l'absorption du glucose produit par l'amylolyse, dans la mesture où d'autres substances réductrices peuvent interférer dans le 
dosage. Ces réserves étant faites, on note que la quantité de sucres réducteurs absorbés au cours des 8 premières heures postprandiales, représente 36 à 43 p. 100 de l'ingéré pour l'orge contre 44 à 48 p. 100 pour le blé (Rerat, VaISSAde \& VaugeLADE, 1979), cependant que les quantités d'amidon évacuées par l'estomac en $7 \mathrm{~h}$ représentent 81,8 p. 100 de l'ingéré pour l'orge contre 86,8 pour le blé. Il y a done concordance puisque 7 à $8 \mathrm{~h}$ après le repas c'est pour le blé que sont enregistrées les quantités cumulées les plus importantes, d'amidon évacué et de sucres réducteurs absorbés. Mais au-delà de cette observation, on remarque l'écart considérable sous une même unité (p. 100 de l'ingéré) entre quantités évacuées et absorbées. Cet écart que l'on retrouve dans la figure 4 pour divers temps postprandiaux, rend compte des glucides alimentaires déjà évacués mais apparemment non encore absorbés.
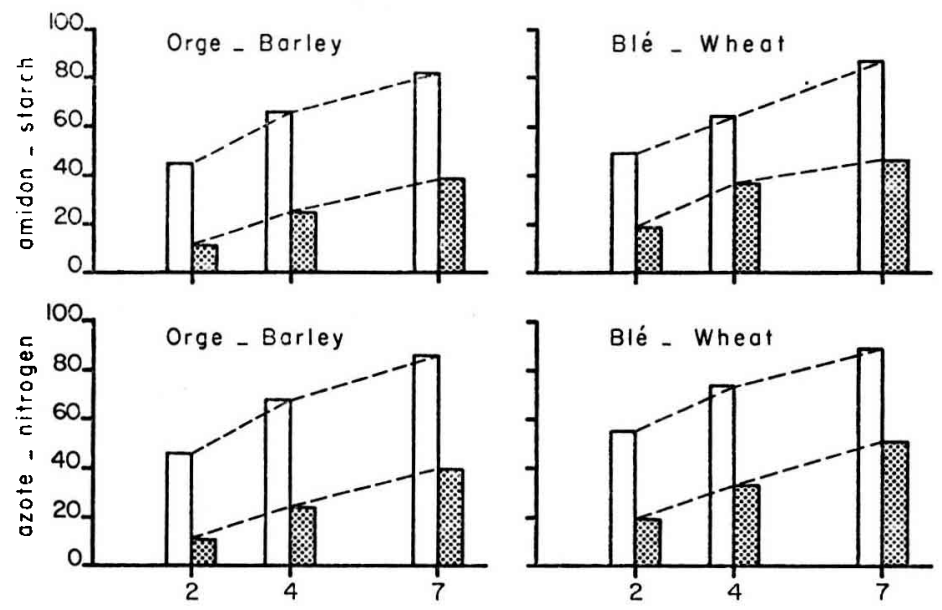

FIG. 4

Quantités évacuées (colonnes blanches, en p. 100 des quantités ingérées) d'amidon ou d'azote après un repas d'orge ou de blé, comparativement aux quantités absorbées (colonnes hâchurées, en p. 100 des quantités ingérées)

de sucres réducteurs ou d'azote a-aminé (RERAT, VAISSADE \& VAUGELAde, 1979).

Emptied quantities (white) of starch or nitrogen after ingestion of either barley or wheat, expressed as per cent of the corresponding intake,

as compared to the respective absorbed quantities (hatched) of reducing sugars

or r-amino nitrogen also expressed as per cent of the corresponding intake, according to Rerat, Vaissade \& Vaugelade (1979).

Une telle différence relève sans doute dans une certaine mesure d'un décalage temporel correspondant à la réalisation des processus d'hydrolyse de l'amidon évacué et de transport du glucose résultant vers le sang. Mais d'autres facteurs doivent également être pris en compte, quoiqu'il soit difficile de les quantifier avec précision. Dans les bilans comparés interviennent des surestimations de la fraction absorbée (glucose provenant de sources autres que l'amidon; substances réductrices autres que le glucose). A côté de ces surestimations, cumulatives, interviennent simultanément, dans les bilans comparés ici, des sous-estimations de la fraction absorbée, également cumulatives. Celles-ci sont liées au fait qu'une partie des sucres absorbés ne peut être retrouvée dans le sang portal en raison des processus de métabolisation par la paroi 
digestive. Le prélèvement de glucose par l'épithélium intestinal serait en effet de l'ordre de 14 à $21 \mathrm{~g} \cdot \mathrm{h}^{-1}$ et la production d'acide lactique de 2,5 à $3,5 \mathrm{~g} \cdot \mathrm{h}^{-1}$ (Rerat, Vaissade, Vaugelade, 1984).

Aux stades intermédiaires de la digestion (fig. 4) l'écart enregistré entre quantités évacuées et quantités absorbées est nécessairement la résultante des 3 facteurs identifiés ci-dessus : décalage temporel inhérent au déroulement des processus de la digestion, surestimations cumulées découlant de la méthodologie mise en œuvre, sousestimations cumulées liées, compte tenu de la méthode utilisée, au fonctionnement du tube digestif lui-même. L'écart résultant enregistré ici (fig. 4) n'est pas stable dans le temps comme c'était le cas pour la digestion d'un amidon de maïs purifié (CubER, LAPLACE \& Villiers, 1980). Après ingestion de blé il équivaut à 30 et 28 p. 100 de l'ingéré respectivement à $2 \mathrm{~h}$ et $4 \mathrm{~h}$, et atteint $41 \mathrm{p} .100$ à la septième heure. Dans le cas de l'orge, il représente 34 p. 100 de l'ingéré après $2 \mathrm{~h}$, puis 40 et 42 p. 100 respectivement à 4 et 7 h. Mais il reste exclu d'évaluer la part sans doute fluctuante de chacun des facteurs responsables de l'écart résultant, qui est de toute façon du même ordre de grandeur pour les 2 céréales après $7 \mathrm{~h}$. De surcroît l'importance globale de l'écart évolue a priori au cours du temps postprandial entre deux cas extrêmes : (i) évacuation massive dans les premières minutes consécutives au repas, alors qu'aucune élévation de la glycémie portale n'est encore décelable; (ii) évacuation achevée, alors que l'absorption se poursuit à partir des matériaux encore présents dans l'intestin grêle. Ceci suggère que l'écart global résultant devrait s'amoindrir dans les étapes terminales de la digestion.

Si l'on procède à un même bilan comparatif après l'achèvement de la digestion, authentifié par l'annulation des différences porto-artérielles, le facteur décalage temporel est alors exclu. L'écart observé est donc la résultante finale des sur- et sousestimations cumulées. Cette fraction non retrouvée au niveau du sang dans un tel bilan d'absorption ressort à 33 p. 100 (blé) et 47 p. 100 (orge) des sucres ingérés (RERAT, 1981). Ces valeurs qui représentent l'écart cumulé en quelques 20 heures de digestion sont analogues (blé) ou peu supérieures (orge) à celles enregistrées au cours des 7 premières heures postprandiales. Ceci témoigne du fait que, au cours de la période postprandiale il y a très certainement à la fois variation de l'importance globale de l'écart entre quantités évacuées et absorbées et variation de la part des facteurs responsables de cet écart, même s'il paraît probable que la métabolisation par la paroi soit prédominante.

En somme, cette tentative d'analyse des liens entre évacuation gastrique de l'amidon et absorption des glucides ne permet pas de savoir si l'évacuation gastrique est ou non limitante de l'absorption. De surcroit, le parallélisme des profils d'évacuation a été établi pour les 7 premières heures postprandiales. Si ce parallélisme se vérifiait sur la totalité de la période d'évacuation, celle-ci devrait être achevée plus tôt pour le blé. On pourrait donc attendre que l'achèvement de la digestion des glucides, attesté par l'annulation des différences porto-artérielles, intervienne également plus tôt pour le blé. Or la digestion des glucides du blé est plus longue d'environ $2 \mathrm{~h}$ par rapport à celle enregistrée pour l'orge (RERAT, 1981). Il est vrai que, sous une même quantité de céréale broyée et dans les conditions de notre expérience, le blé apporte 17 p. 100 d'amidon de plus que l'orge. Enfin, la différence importante entre les coefficients d'absorption des sucres réducteurs au terme de la digestion (blé 67 p. 100 ; orge 53 p. 100) suggère que, à des facteurs tels que l'ampleur de la métabolisation, s'ajoute peut être une influence de la digestibilité i.e. accessibilité ou aptitude de l'amidon de ces céréales à l'hydrolyse enzymatique. On ne peut donc en définitive 
que dresser un bilan en forme de constat : Pour une même quantité ingérée de céréale broyée, et par rapport à l'orge, la consommation de blé conduit à ingérer 17 p. 100 d'amidon de plus, à récupérer par absorption de sucres réducteurs 14 p. 100 de l'ingéré total en plus, le tout dans un temps total de digestion plus long de $2 \mathrm{~h}$ avec le concours d'une évacuation gastrique et corrélativement d'une absorption plus importantes aux temps intermédiaires.

\section{Evacuation gastrique de l'azote et digestion des matières azotées de l'orge et du blé}

Une procédure analogue de comparaison peut être mise en œuvre pour ce qui concerne les matières azotées. Sur ce point, l'écart entre les 2 céréales est moindre que pour les glucides puisque à quantité égale de céréale broyée fraîche, le blé apporte environ 9 p. 100 d'azote de plus que l'orge. C'est encore pour le blé que l'évacuation gastrique de l'azote est la plus précoce et la plus importante en termes de bilan au cours des 7 premières heures postprandiales. Si l'on rapproche ces observations des bilans d'absorption apparente d'azote $\alpha$-aminé (Rerat, Vaissade \& Vaugelade, 1979 ; RERAT, 1981) on vérifie la correspondance entre évacuation plus importante de l'azote et absorption plus intense d'acides aminés dans le cas du blé. Là encore un tel rapprochement constitue une approximation dans la mesure où sont pris en compte comparativement l'azote Kjeldahl de la céréale, l'azote Kjeldahl de contenus digestifs où se retrouvent à la fois les protéines de la céréale et les protéines endogènes, et enfin l'azote $\alpha$-aminé dans le sang après absorption des produits d'hydrolyse des protéines exogènes et endogènes. L'interférence des protéines endogènes constitue du reste une difficulté particulière dans la mesure où toute expression en pourcentage de l'ingéré est en fait l'expression d'une équivalence à un pourcentage de l'ingéré. Sous ces réserves, la différence d'évacuation de l'azote entre les 2 céréales, importante dès les 30 premières minutes postprandiales, se solde par un coefficient d'absorption de l'azote $\alpha$-aminé en 8 h de 53 à 56 p. 100 pour le blé contre 35 à 43 p. 100 dans le cas de l'orge (Rerat, Vaissade \& Vaugelade, 1979). Mais, comme pour les glucides et quelle que soit la céréale, et en dépit de l'absorption fort probable d'azote endogène en plus de l'azote d'origine alimentaire, il existe en termes de pourcentage de l'ingéré un retard important de l'absorption par rapport à l'évacuation (fig. 4). Cet écart pourrait, toujours sous les réserves précitées, relever de 2 causes : d'une part excrétion fécale d'une certaine quantité de matières azotées, et d'autre part prélèvement d'azote par la paroi au moins aussi important que celui du glucose. L'amplitude du décalage entre évacuation et absorption est variable : elle équivaut pour l'orge à 36,44 et 47 p. 100 de l'ingéré respectivement à 2,4 et $7 \mathrm{~h}$, et pour le blé à 36 , 41 et 38 p. 100 de l'ingéré aux mêmes temps. Comme pour les glucides, ces valeurs sont peu différentes de celles de la fraction non retrouvée dans un bilan d'absorption d'azote $\alpha$-aminé en $24 \mathrm{~h}$, donc établi après achèvement de la digestion : environ 30 p. 100 de l'ingéré ne sont pas retrouvés dans le cas du blé et près de 40 p. 100 dans le cas de l'orge (Rerat, 1981). Par conséquent, l'écart entre proportions évacuées et absorbées, tel qu'il apparaît dans la figure 4 peut encore rendre compte à la fois de la fraction non absorbée (excrétion fécale) et de la métabolisation dans la paroi. En somme, pour une même quantité de céréale broyée ingérée, en prenant encore comme base les résultats enregistrés pour l'orge, la consommation de blé conduit à ingérer 9 p. 100 d'azote en plus, à récupérer par absorption 9 p. 100 
de l'ingéré azoté en plus, le tout dans un moindre temps total de digestion avec le concours d'une évacuation gastrique et corrélativement d'une absorption plus importantes aux temps intermédiaires.

\section{Conclusions}

De ce travail, on peut retenir :

- que la méthodologie fondée sur l'usage de la fistule gastrique chronique est applicable à des régimes autres que purifiés, en l'occurrence de l'orge et du blé broyés ;

- que l'évacuation gastrique du blé est, au cours des 7 heures postprandiales, plus importante que celle de l'orge, l'écart étant établi très précocement puis préservé si l'on en juge par le parallélisme des profils d'évacuations;

- que l'évacuation gastrique de l'amidon et des matières azotées de ces deux céréales est globablement déterminante de l'absorption des sucres réducteurs et des acides aminés, mais qu'il n'est pas possible de préciser si l'évacuation est ou non limitante de l'absorption.

\section{Remerciements}

A Sylvie Giger et Michelle Dorleans (Chaire de Zootechnie, Institut National Agronomique, Paris) qui ont bien voulu doser les constituants pariétaux de nos matières premières.

\section{Summary \\ Gastric emptying of barley and wheat in the pig}

Gastric emptying of ground barley and wheat, only admixed with minerals and vitamins ( 4 p. 100 of the diet), was studied in 10 pigs of $49 \mathrm{~kg}$ live weight fitted with a permanent gastric cannula. The kinetic study was made by collecting residual gastric contents after the meal at $30 \mathrm{~min}, 1,2,4$ or $7 \mathrm{~h}$. Dry matter, starch and nitrogen were determined on wet digesta. Three kinetics were established per pig with one test per day. In the case of ground cereals, the validity of this methodology was determined by comparison (fig. 2) with results obtained after slaughter (12 additional pigs) $2 \mathrm{~h}$ after the meal.

The residual gastric amounts were always larger for barley, whatever the variable checked (tables 3 and 4). In the absence of any other significant effect, the multivariate analysis confirmed that gastric emptying of wheat was larger than that of barley (fig. 3). According to the curves (polynomial adjustments) this difference occurred early where after the kinetics of emptying between $30 \mathrm{~min}$. and $7 \mathrm{~h}$ were the same for the 2 cereals (parallelism of curves). The factor responsible for this early effect cannot be identified a priori. Results of gastric emptying of starch and nitrogen were compared (fig. 4) with data of the literature concerning the absorption of carbohydrates and amino acids derived from the digestion of barley and wheat. It appeared that the gastric emptying was highly responsible for the rate of absorption of reducing sugars and amino acids; however it was not possible to establish whether the emptying was absorption limiting or not.

Key words : Gastric emptying, cereals, pig, proteins, carbohydrates. 


\section{Références bibliographiques}

Buraczewska L., Zebrowska T., Wünsche J., Hennig U., Krawielitzki K., Kreienbring F., Meinl M., Borgmann E., Bock H.D., 1979. Untersuchungen über die Proteinverdaulichkeit und Aminosaürenresorption in verschiedenen Abschnitten des Verdauungstraktes beim Schwein. 4) Rohprotein- und Aminosaürenverdaulichkeit sowie Passagerate in Duodenum, Ileum und Gesamt-Verdauungstrakt wachsender Schweine. Arch. Tierernähr., 29, 437-460.

Cuber J.C., Kaboré Ch., Laplace J.P., 1981. Evacuation gastrique comparée de l'orge et du blé broyés chez le porc (Abstr.). Reprod. Nutr. Dévelop., 21, 773.

Cuber J.C., Laplace J.P., 1979. Evacuation gastrique de l'amidon et de l'azote d'un régime semi-purifié à base d'amidon de maïs chez le porc. Ann. Zootech., 28, 173-184.

Cuber J.C., Laplace J.P., Villiers P.A., 1980. Fistulation de l'estomac et contenus gastriques résiduels après ingestion d'un régime semi-purifié à base d'amidon de maïs chez le porc. Reprod. Nutr. Dévelop., 20, 1161-1172.

Henry Y., 1968. Utilisation comparée des céréales comme seuls aliments du porc pendant la période de finition. Ann. Zootech., 17, 183-197.

Henry Y., Rerat A., 1966. Utilisation des pommes de terre déshydratées et fraîches dans l'alimentation du porc en croissance en comparaison avec l'orge. Ann. Zootech., 15, 231-251.

Holz F., 1977. Automatische, enzymatisch-photometrische Bestimmung des Stärkegehaltes von Cerealien. Landwirtsch. Forsch., 33, 228-249.

IVAN M., FARrell D.J., 1976. Nutritional evaluation of wheat. 5) Disappearance of components in digesta of pigs prepared with two reentrant cannulae. Anim. Prod., 23, $111-119$.

Laplace J.P., Pons O., Cuber J.C., Kaboré Ch., 1984. Evacuation gastrique et composition en acides aminés des protéines du régime chez le porc. Ann. Zootech., 33, 59-72.

Laplace J.P., Pons O., Simon P., 1983. Révision des effets de l'amidon et des protéines du régime sur l'évacuation gastrique chez le porc. Ann. Zootech., 32, 507-524.

Low A.G., 1979 a. Studies on digestion and absorption in the intestines of growing pigs. 5) Measurements of the flow of nitrogen. Br. J. Nutr., 41, 137-146.

Low A.G., 1979 b. Studies on digestion and absorption in the intestines of growing pigs. 6) Measurements of the flow of amino acids. Br. J. Nutr., 41, 147-156.

PoтthofF R.F., RoY S.N., 1964. A generalized multivariate analysis of variance model useful especially for growth curve problems. Biometrika, 51, 313-326.

Rerat A., 1981. Chronologie et bilans de l'absorption des sucres réducteurs et de l'azote $\alpha$-aminé chez le porc selon la nature de l'aliment. Bull. Acad. Nat. Med., 165, 11311137.

Rerat A., Vaissade P., Vaugelade P., 1979. Absorption kinetics of amino acids and reducing sugars during digestion of barley or wheat meals in the pig : preliminary data. Ann. Biol. anim. Biochim. Biophys., 19, 739-747.

Rerat A.A., Vaissade P., Vaugelade P., 1984. Absorption kinetics of some carbohydrates in conscious pigs. 2) Quantitative aspects. Br. J. Nutr., 51, 517-529.

Sauer W.C., Kennelly J.J., Aherne F.X., Cichon R.M., 1981. Availabilities of amino acids in barley and wheat for growing pigs. Can. J. anim. Sci., 61, 793-802.

Sauer W.C., Stothers S.C., Phillips G.D., 1977. Apparent availabilities of amino acids in corn, wheat, and barley for growing pigs. Can. J. anim. Sci., 57, 585-597.

VAN SOEST P.J., 1963 a. Use of detergents in the analysis of fibrous feeds. 1) Preparation of fiber residue of low nitrogen content. J. Ass. off. analyt. Chem., 46, 825-829.

VAN Soest P.J., 1963 b. Use of detergents in the analysis of fibrous feed. 2) A rapid method for the determinations of fiber and lignin. J. Ass. off. analyt. Chent., 46, 829835. 